\title{
rAd5 prime/NYVAC-B boost regimen is superior to NYVAC-B prime/rAd5 boost regimen for both response rates and magnitude of CD4 and CD8 T-cell responses
}

P Bart ${ }^{*}$, Y Huang ${ }^{2}$, N Frahm ${ }^{3}$, S Karuna ${ }^{3}$, M Allen ${ }^{4}$, NK Kochar ${ }^{3}$, S Chappuis ${ }^{1}$, J Gaillard ${ }^{1}$, B Graham ${ }^{5}$, G Pantaleo ${ }^{1}$

From AIDS Vaccine 2012

Boston, MA, USA. 9-12 September 2012

\section{Background}

HVTN 078 is a randomized, double blind phase 1b clinical trial to evaluate the safety and immunogenicity of heterologous prime/boost vaccine regimens (NYVAC-B/ rAd5 vs. rAd5/NYVAC-B) in healthy, HIV-1 uninfected, Ad5 seronegative adult participants.

\section{Methods}

The rAd5 vaccine expressed clade B Gag-Pol and the gp140 of HIV-1 92RW020 (clade A), HxB2/Bal-V3/ $\triangle$ V1V2 (clade B) and 97ZA012 (clade C). The NYVAC-B vaccine expressed clade B Gag-Pol-Nef and the gp120 of Bx08 (clade B). 80 healthy, HIV-1 uninfected, Ad5 seronegative volunteers, aged 18 to 45 years, were randomized to the placebo arm $(\mathrm{n}=5)$ or one of 4 treatment $(\mathrm{T})$ arms: $\mathrm{T} 1$ $(\mathrm{n}=30), 2 x N Y V A C-\mathrm{B} / 1 \mathrm{xrAd} 5$ (10E10); T2 $(\mathrm{n}=15), 1 \mathrm{xrAd} 5$ (10E8)/2xNYVAC-B; T3 (n=15), $1 \times$ rAd5 (10E9)/2xNYVAC-B; T4 ( $n=15), 1 x r A d 5$ (10E10)/2xNYVAC-B.

Intracellular cytokine staining responses (percent of $\mathrm{CD} 4+$ and CD8+ T cells producing IFN- $\gamma$ and/or IL-2 in response to stimulation with global PTE peptides) were assessed two weeks after the final vaccination.

\section{Results}

For CD4+ T cells, the overall response rates for IFN- $\gamma$ and/or IL- 2 among the vaccinees were $42.9 \%, 93.3 \%$, 92.3\%, and $85.7 \%$ for $\mathrm{T} 1-\mathrm{T} 4$, respectively; and the median response magnitudes for positive responders were $0.26 \%$, $0.76 \%, 0.40 \%$, and $0.76 \%$ for $\mathrm{T} 1-\mathrm{T} 4$, respectively. Both response rates $(\mathrm{p}<0.01)$ and magnitudes $(\mathrm{p}<0.03)$ of $\mathrm{CD} 4+$
$\mathrm{T}$-cell responses were significantly lower in $\mathrm{T} 1$ compared to the other three treatment groups. For CD8+ T cells, the overall response rates were $65.5 \%, 73.3 \%, 76.9 \%$ and $85.7 \%$ for T1-T4, respectively; and median response magnitudes for positive responders were $0.32 \%, 0.99 \%, 1.86 \%$, and $1.65 \%$, respectively. Response rates were not significantly different between groups; however, response magnitudes were significantly lower in $\mathrm{T} 1$ compared to the other three arms $(\mathrm{p}<0.04)$.

\section{Conclusion}

Priming with rAd5 followed by NYVAC-B boost is superior to priming with NYVAC-B followed by rAd5 boost for both response rates and the magnitude of $\mathrm{CD} 4+$ and CD8+ T-cell responses.

\section{Author details}

${ }^{1}$ CHUV, Lausanne, Switzerland. ${ }^{2}$ SCHARP, Seattle, WA, USA. ${ }^{3} \mathrm{HVTN}$, Seattle, WA, USA. ${ }^{4}$ DAIDS, NIAID, NIH, Bethesda, MD, USA. ${ }^{5}$ Vaccine Research Center, NIAID, NIH, Bethesda, MD, USA.

Published: 13 September 2012 